\section{CORRECTION}

View Article Online

View Journal I View Issue

\title{
Correction: Live cell single molecule tracking and localization microscopy of bioorthogonally labeled plasma membrane proteins
}

Cite this: Nanoscale, 2020, 12, 18476

\author{
Andres I. König, ${ }^{\text {a,b }}$ Raya Sorkin, ${ }^{c}$ Ariel Alon, ${ }^{a, b}$ Dikla Nachmias, ${ }^{\text {a,b }}$ Kalyan Dhara, ${ }^{d}$ \\ Guy Brand, ${ }^{c}$ Ofer Yifrach, ${ }^{a}$ Eyal Arbely, (iD a,b,d Yael Roichman ${ }^{c, e}$ and Natalie Elia (D) *a,b
}

DOI: $10.1039 / d 0 n r 90195 d$

Correction for 'Live cell single molecule tracking and localization microscopy of bioorthogonally labeled plasma membrane proteins' by Andres I. König et al., Nanoscale, 2020, 12, 3236-3248, DOI: 10.1039/ C9NR08594G.

The authors regret that in the original manuscript, position 128 in the EGFR was referred to incorrectly as L128, rather than N128. This error occurred on page 3237 in the following sentence: "To this end, EGFR was mutated to carry a TAG codon at the previously published labeling site, Leu 128, and cloned into a single expression vector that encodes the cognate pair of tRNA cua: tRNA-synthetase ${ }^{17,18}$ (Fig. S1a†)."

The error also occurred in Fig. 1a, the corrected version of which is displayed below, along with the original, unaltered caption. These errors do not affect any of the experimental results and discussion or conclusions reported in the paper, only the display of the figure and text.

The Royal Society of Chemistry apologises for these errors and any consequent inconvenience to authors and readers.

\footnotetext{
${ }^{a}$ Department of Life Sciences, Ben-Gurion University of the Negev, Beer Sheva 84105, Israel. E-mail: elianat@post.bgu.ac.il; Fax: +972-8-6477546; Tel: +972-8-6428735 ${ }^{b}$ National Institute for Biotechnology in the Negev (NIBN), Ben-Gurion University of the Negev, Beer Sheva 84105, Israel

${ }^{c}$ Raymond \& Beverly Sackler School of Chemistry, Tel Aviv University, Tel Aviv 6997801, Israel

${ }^{d}$ Department of Chemistry, Ben-Gurion University of the Negev, Beer Sheva 84105, Israel

${ }^{e}$ Raymond \& Beverly Sackler School of Physics, and the Center for light-matter interaction, Tel Aviv University, Tel Aviv 6997801, Israel
} 
a

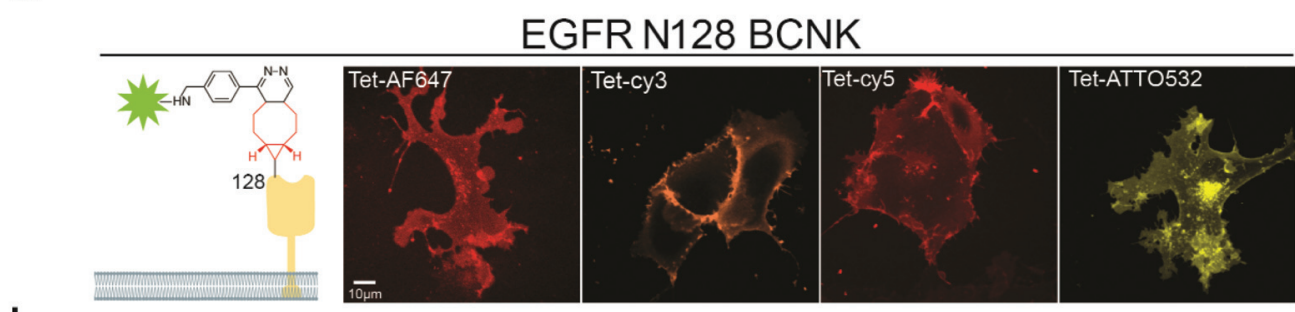

b
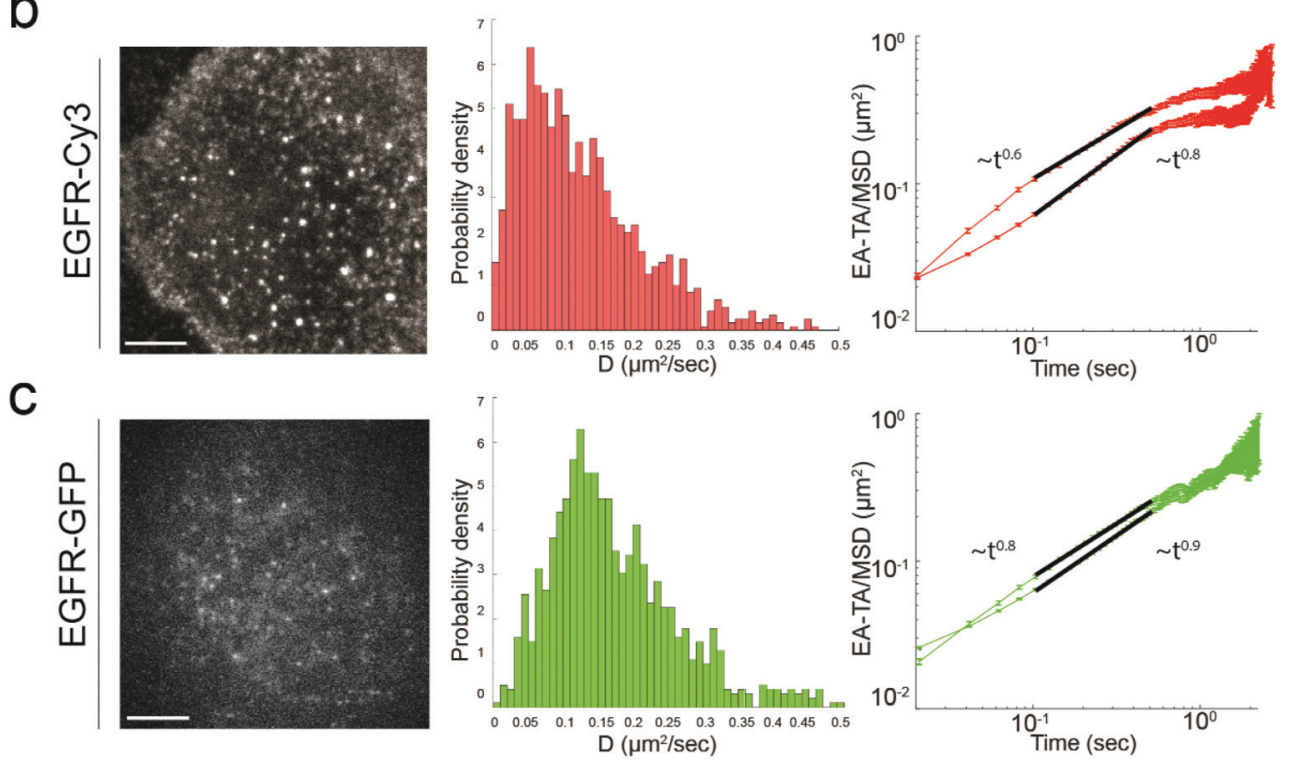

d

e

f
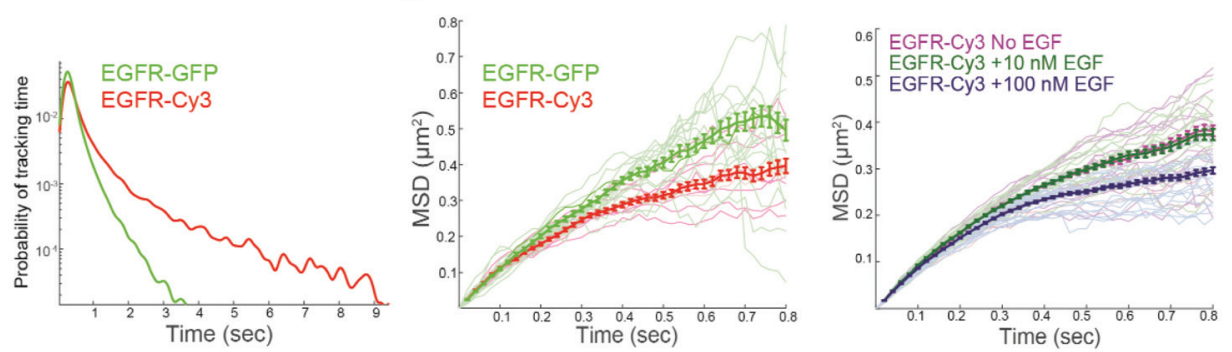

Fig. 1 Bioorthogonal labeling with Fl-dyes enables single particle tracking of EGFR. (a) Left panel: Schematic representation of the labeling strategy. The extracellular domain of EGFR (yellow) was mutated to carry the ncAA BCNK (red) at position 128. Labeling was obtained via a bioorthogonal reaction between $\mathrm{BCNK}$ and a tetrazine conjugated Fl-dye (green star). Panels 2-5: Live COS7 cells expressing EGFR ${ }^{128 B C N K}$ together with GCE components (Fig. S1a †) were labeled with $1.5 \mu$ M of: Tet-AF647, Tet-Cy3, Tet-Cy5 or Tet-ATTO532 (Fig. S1b $\dagger$ ) and imaged using a spinning disk confocal microscope. Shown are single slices taken from the center of the cell. Scale bar $=10 \mu \mathrm{m}$. (b and c) COS7 cells transfected with either GCE system plasmid with carrying EGFR ${ }^{128 B C N K}$ and labeled with Tet-Cy3 (b) or EGFR-GFP (c), were imaged in TIRF mode at $50 \mathrm{fps}$. Tracks were then obtained as described in methods section. Left panel: Representative images of the first frame (the complete video sequences are provided in Videos 1 and 2). Middle panels: The diffusion coefficient $[D]$ of all particles tracked. EGFR-Cy3 median, $0.11 \mu \mathrm{m}^{2} \mathrm{~s}^{-1}$ (with $95 \%$ confidence interval of $0.02-0.4 \mu \mathrm{m}^{2}$ $\mathrm{s}^{-1}, n=1177$ ). EGFR-GFP median, $0.15 \mu \mathrm{m}^{2} \mathrm{~s}^{-1}$ (with $95 \%$ confidence interval of $0.04-0.47 \mu \mathrm{m}^{2} \mathrm{~s}^{-1}, n=1018$ ). Right panels: Ensemble-averaged MSD (top plots) and ensemble-averaged time-averaged MSD (bottom plots) in log scale, MSD $\sim t^{\alpha}$, where $\alpha$ is the anomalous exponent. (d) The tracking time probability density function of EGFR-GFP (green) or EGFR-Cy3 (red). (e) MSD curves of EGFR-GFP (green) or EGFR-Cy3 (red) as a function of time. Thick lines are the ensemble means for each labeling type with error bars representing SEM. Thin lines represent measurements obtained from individual cells (EGFR-Cy3 $n=9$ cells, EGFR-GFP $n=17$ cells, obtained from three independent experiments). (f) MSD curves obtained for EGFR-Cy3 under three different concentrations of EGF: no EGF (magenta), 10 nM EGF (dark green) or 100 nM EGF (blue) as a function of time. Thick lines are the ensemble means for each labeling type with error bars representing SEM. Thin lines represent measurements obtained from individual cells ( $\mathrm{no}$ EGF $n=18$ cells, $10 \mathrm{nM} \mathrm{EGF} n=15$ cells, and $100 \mathrm{nM} \mathrm{EGF} n=18$ cells). 